\title{
Níveis de Ansiedade e Depressão entre Residentes de Pediatria
}

\section{Anxiety and Depression Levels among Resident Pediatricians}

\author{
Luciano Garcia Lourençãol \\ Priscila Regina Teixeiral \\ Cláudia Eli Gazetta II \\ Maria Helena Pinto II \\ Elizangela Gianini Gonsalez" \\ Daniela Salvagni RottaII
}

\section{PALAVRAS-CHAVE}

- Internato e Residência.

- Ansiedade.

- Depressão.
REVISTA BRASILEIRA DE EDUCAÇÃO MÉDICA
${ }^{I}$ Universidade Federal do Rio Grande. Rio Grande, RS, Brasil.

${ }^{\text {II }}$ Faculdade de Medicina de São José do Rio Preto, São José do Rio Preto, SP, Brasil.
Objetivo: Avaliar os níveis de ansiedade e depressão dos profissionais matriculados em um Programa de Residência Médica em Pediatria de uma instituição de ensino do interior do Estado de São Paulo. Métodos: Estudo transversal descritivo, com todos os médicos residentes matriculados no Programa de Residência Médica em Pediatria. Os dados foram coletados entre os meses de novembro de 2013 e fevereiro de 2014, utilizando-se três instrumentos autoaplicáveis: um instrumento elaborado pelos autores, com dados sociodemográficos; a Escala de Ansiedade de Beck ou Inventário de Ansiedade de Beck e a Escala de Depressão de Beck ou Inventário de Depressão de Beck. Os níveis de ansiedade e depressão foram analisados por uma psicóloga segundo dados dos instrumentos e categorizados em ausência de depressão/ansiedade, depressão/ansiedade leve, depressão/ansiedade moderada e depressão/ ansiedade grave. Resultados: Participaram do estudo 36 médicos residentes. Houve predominância do sexo feminino (91,4\%), idade mediana de 28 anos (mínimo: 25; máximo: 34), solteiros (86,11\%), renda familiar de dez ou mais salários mínimos $(47,1 \%)$, jornada de trabalho de 12 horas ou mais $(55,6 \%)$, sem atividade física $(55,5 \%)$ e de lazer $(44,2 \%)$, com outro vínculo laboral $(71,4 \%)$, satisfeitos com o trabalho (88,9\%); 52,8\% pensaram em desistir do programa. Ansiedade esteve presente em $50,0 \%$ dos profissionais e depressão em $44,4 \%$. Houve associação estatística da ansiedade com a faixa etária $(p<0,005)$ e com o desejo de desistir do programa $(p=0,038)$; e da depressão com a faixa etária ( $p=0,001)$, prática de atividade física $(p=0,016)$, atividades de lazer $(p=0,012)$ e com o desejo de desistir do programa $(p=0,008)$. Conclusão: Os niveis de ansiedade e depressão foram superiores aos observados em outros programas, havendo associação destes transtornos com a faixa etária e ausência de atividade física e de lazer, evidenciando a necessidade de maior atenção e suporte aos profissionais, de implementação de ações de controle dos fatores estressores entre os residentes de Pediatria ede estratégias de promoção do bem-estar físico e mental desses profissionais. 


\section{KEYWORDS}

- Internship and Residency.

- Anxiety.

- Depression.

Recebido em: 03/01/2017

Aprovado em: 09/08/2017

\section{ABSTRACT}

Objective: To evaluate the levels of anxiety and depression among professionals enrolled in a Pediatric Residency Program of an educational institution in the State of São Paulo, Brazil. Methods: Cross-sectional and descriptive study with all, registered residents at a pediatric residency program. The data were collected between the months of November 2013 and February 2014, using three instruments: an instrument drawn up by the authors, containing demographic data; the Beck anxiety Scale or Beck anxiety inventory and the Beck Depression scale or Beck Depression inventory. Depression anxiety levels were evaluated by a psychologist, according to data produced by the instruments and categorized in to four levels: absence of depression/anxiety, mild depression/anxiety, moderate depression/anxiety and severe depression/anxiety. Results: The study was based on a sample of 36 medical residents. There was a predominance of females $(91.4 \%)$, with a median age of 28 years (minimum: 25; maximum: 34), single (86.11\%), with a household income of 10 minimum monthly wages or more (47.1\%), a working day of 12 hours or more (55.6\%), not regularly engaged in physical activity (55.5\%) and leisure (44.2\%), with another job (71.4\%) satisfied with their job (88.9\%) and having thought about quitting the program (52.8\%). Anxiety was present in $50.0 \%$ and depression in $44.4 \%$. Statistical associations were found between anxiety and the age group $(p<0.005)$ and with a desire to give up the program ( $p=0.038)$; and between depression and age group ( $p=0.001)$, practice of physical activity $(p=0.016)$, leisure activities $(p=0.012)$ and a desire to give up the program ( $p$ $=0.008)$. Conclusion: The levels of anxiety and depression were higher than those observed in other programs, with associations observed between these disorders and age, lack of physical activity and leisure, highlighting the need for greater attention and professional support, and the implementation of control measures for stressor factors among the resident pediatricians, and of strategies to promote the physical and mental well-being of these professionals.

\section{INTRODUÇÃO}

Os primeiros programas de residência médica tiveram início em 1890, no Hospital Johns Hopkins, nos Estados Unidos ${ }^{1}$. No Brasil, a residência médica foi normatizada em 1964 pela Associação Brasileira de Educação Médica (Abem), e atualmentehá 3.500 programas distribuídos em 53 especialidades reconhecidas pelo Conselho Federal de Medicina (CFM) $)^{1,2}$.

Embora seja considerada uma forma eficiente de capacitação profissional, a residência médica é uma experiência de trabalho intenso e exaustivo, tornandoos residentes um grupo de risco para o desenvolvimento de distúrbios emocionais e disfunções profissionais, com alta incidência de ansiedade e depressão ${ }^{3-5}$.

O desgaste emocional decorrido das relações dos médicos residentes com o ambiente de trabalho é um importante fator na determinação de transtornos como ansiedade e depressão. Esses transtornos desencadeiam um emaranhado de sensações e alterações comportamentais, culminando com a perda de interesse pelas atividades profissionais e do prazer nas relações interpessoais, baixa autoestima, desesperança, sensação de falta de energia, dificuldade de concen- tração, além de manifestações físicas como cefaleias, distúrbios de sono e apetite, náuseas, dores musculares e perda da libido ${ }^{4,6,7}$.

Estudos apontam que os desgastes inerentes às atividades assistenciais, a insegurança, o excesso de trabalho, a falta de supervisão e a baixa remuneração, associados à má gestão do sistema de saúde brasileiro, que sobrecarrega os serviços e os profissionais, contribuem para o adoecimento psíquico dos residentes médicos ${ }^{4,8,9}$.

Considerando as angústias e os comportamentos negativos vivenciados pelos médicos residentes, que comprometem o desempenho profissional durante o processo de formação e treinamento em serviço, incluindo aqueles em formação em Pediatria, conhecer os níveis de ansiedade e depressão desses profissionais pode contribuir para o direcionamento de ações de melhorias dos programas de residência e de promoção da saúde física e mental desses profissionais.

Ante o exposto, o objetivo deste estudo foi avaliar os níveis de ansiedade e depressão dos profissionais matriculados em um Programa de Residência Médica em Pediatria de uma instituição de ensino do interior do Estado de São Paulo. 


\section{MÉTODOS}

Estudo transversal descritivo, de base populacional, realizado entre os profissionais matriculados no Programa de Residência Médica em Pediatria de uma instituição de ensino do interior do Estado de São Paulo no ano de 2013 que consentiram em participar da pesquisa após terem sido informados sobre os objetivos e finalidades do estudo, totalizando 36 profissionais. Foram excluídos aqueles que estavam afastados das atividades profissionais por qualquer motivo durante o período de coleta dos dados.

A coleta de dados foi realizada com três instrumentos autoaplicáveis: um instrumento elaborado pelos autores, contendo dados pessoais, como idade, sexo, estado civil, categoria profissional, renda familiar e se está satisfeito com o programa ou pensou em desistir dele; a Escala de Ansiedade de Beck ou Inventário de Ansiedade de Beck, que mede a severidade dos sintomas de ansiedade do indivíduo por meio de 21 questões sobre como tem se sentido na última semana, expressas em sintomas comuns de ansiedade com quatro possíveis respostas (ausência; levemente; moderadamente; severamente) ${ }^{10}$; e a Escala de Depressão de Beck ou Inventário de Depressão de Beck, composto também por 21 questões que abordam itens relacionados a sintomas depressivos, como desesperança, irritabilidade e cognições, culpa ou sentimento de estar sendo punido, e sintomas físicos como fadiga, perda de peso e diminuição da libido ${ }^{11}$.

Os dados demográficos e socioeconômicos caracterizaram a população do estudo. Os níveis de ansiedade e depressão foram analisados por uma psicóloga segundo dados dos instrumentos e categorizados em ausência de depressão/ansiedade, depressão/ansiedade leve, depressão/ansiedade moderada e depressão/ansiedade grave.

A análise dos dados foi realizada com uso do programa Statistical Package for Social Sciences, versão 17.0. Comparações foram feitas pelo teste de concordância de Kappa, considerado significante um valor-p inferior ou igual a 0,05.

Respeitando as exigências formais contidas nas normas nacionais e internacionais regulamentadoras de pesquisas que envolvem seres humanos, este estudo foi submetido à apreciação do Comitê de Ética em Pesquisa da Faculdade de Medicina de São José do Rio Preto e aprovado em 12 de novembro de 2013, com o Parecer no ${ }^{460.331 . ~}$

\section{RESULTADOS}

Participaram do estudo 36 médicos residentes, sendo a maioria do sexo feminino (91,4\%). A idade dos profissionais variou de 24 a 35 anos, com média de 27,1 anos (DP: $\pm 2,2$ anos) e IC $95 \%$ de 27,9 a 26,3 anos; solteiros $(85,7 \%)$, com renda fa- miliar acima de dez salários mínimos (47,1\%); a maioria dos profissionais trabalhava 12 horas diárias ou mais (55,6\%), não praticava atividade física $(55,5 \%)$ e referiu atividade de lazer $(55,8 \%)$. Destaca-se que $71,4 \%$ dos profissionais referiram ter outro vínculo profissional; 88,9\% afirmaram estar satisfeitos com o programa de residência, apesar de 52,8\% deles já terem pensado em desistir do curso.

Conforme observado na Tabela 1, 50,0\% dos profissionais apresentaram ansiedade, sendo $20,0 \%$ ansiedade moderada e $2,9 \%$ ansiedade grave. A análise das características sociodemográficas e de satisfação dos médicos residentes mostrou que os profissionais com ansiedade eram prevalentemente do sexo feminino (42,9\%), na faixa etária de 26 a 30 anos $(34,4 \%)$, solteiros $(42,9 \%)$, não praticavam atividade física $(36,2 \%)$, não tinham atividades de lazer (32,3\%), trabalhavam 12 horas diárias ou mais $(33,3 \%)$, tinham outro trabalho $(28,6 \%)$, renda de dez salários mínimos ou mais $(26,5 \%)$, estavam satisfeitos com o programa de residência $(41,6 \%)$ e já haviam pensado em desistir do programa (36,1\%). Houve associação estatística da ansiedade com a faixa etária $(\mathrm{p}<0,005)$ e com o desejo de desistir do programa $(p=0,038)$.

A prevalência de depressão entre os residentes de Pediatria foi de 44,4\%. Em relação às características sociodemográficas e de satisfação dos profissionais com depressão, observou-se que eram do sexo feminino $(42,9 \%)$, na faixa etária de 26 a 30 anos (31,3\%), solteiros (34,3\%), não praticavam atividade física $(36,1 \%)$, não tinham atividades de lazer (32,3\%), trabalhavam 12 horas diárias ou mais $(27,7 \%)$, tinham outro trabalho $(28,6 \%)$, renda de dez salários mínimos ou mais $(26,5 \%)$, estavam satisfeitos com o programa de residência $(41,6 \%)$ e já pensaram em desistir do programa $(36,1 \%)$. Houve associação estatística da depressão com a faixa etária $(\mathrm{p}=0,001)$, prática de atividade física $(\mathrm{p}=0,016)$, atividades de lazer $(\mathrm{p}=0,012)$ e com o desejo de desistir do programa $(\mathrm{p}=0,008)$ (Tabela 2$)$.

A Tabela 3 mostra a relação entre ansiedade e depressão para os médicos residentes, evidenciando que $50,0 \%$ dos profissionais apresentaram sintomas de ansiedade associada ou não à depressão. Houve concordância razoável entre profissionais com ausência de ansiedade e depressão ( $K=0,378)$, e significância estatística na concordância entre profissionais com ansiedade e depressão grave $(p=0,003)$.

\section{DISCUSSÃO}

O perfil dos residentes de Pediatria observado neste estudo é semelhante ao relatado em outros estudos, apontando prevalência de profissionais do sexo feminino ${ }^{12}$, solteiros e com idade inferior a $30 \operatorname{anos}^{9,12-14}$. 


\section{TABELA 1}

Distribuição dos níveis de ansiedade segundo características sociodemográficas e de satisfação dos médicos residentes. São José do Rio Preto, 2013-2014

\begin{tabular}{lccccc} 
& \multicolumn{4}{c}{ Ansiedade } & \multirow{2}{*}{ Total } \\
\cline { 2 - 5 } & Ausência & Leve & Moderada & Grave & \\
Sexo $(\mathrm{p}=0,377)$ & $\mathrm{n}(\%)$ & $\mathrm{n}(\%)$ & $\mathrm{n}(\%)$ & $\mathrm{n}(\%)$ & $\mathrm{n}(\%)$ \\
Masculino & $1(2,9)$ & $2(5,7)$ & - & - & $3(8,6)$ \\
Feminino & $17(48,5)$ & $7(20,0)$ & $7(20,0)$ & $1(2,9)$ & $32(91,4)$ \\
Total & $18(51,4)$ & $9(25,7)$ & $7(20,0)$ & $1(2,9)$ & $35^{*}(100,0)$
\end{tabular}

Faixa etária $(\mathrm{p}<0,005)$

$\begin{array}{lccccc}20-25 \text { anos } & 5(15,7) & 2(6,2) & 2(6,2) & - & 9(28,1) \\ 26-30 \text { anos } & 11(34,4) & 7(21,9) & 4(12,5) & - & 22(68,8) \\ 31-35 \text { anos } & - & - & - & 1(3,1) & 1(3,1) \\ \text { Total } & 16(50,0) & 9(28,1) & 6(18,7) & 1(3,1) & 32^{*}(100,0) \\ \text { Estado civil }(\mathrm{p}=0,598) & & & & & \\ \text { Casado } & 3(8,6) & 2(5,7) & - & - & 5(14,3) \\ \text { Solteiro } & 15(42,8) & 7(20,0) & 7(20,0) & 1(2,9) & 30(85,7) \\ \text { Total } & 18(51,4) & 9(25,7) & 7(20,0) & 1(2,9) & 35^{*}(100,0)\end{array}$

Atividade física $(\mathrm{p}=0,212)$

$\begin{array}{lccccc}\text { Sim } & 11(30,6) & 3(8,3) & 2(5,6) & - & 16(44,5) \\ \text { Não } & 7(19,4) & 6(16,7) & 6(16,7) & 1(2,8) & 20(55,5) \\ \text { Total } & 18(50,0) & 9(25,0) & 8(22,3) & 1(2,8) & 36(100,0)\end{array}$

Atividades de lazer $(\mathrm{p}=0,094)$

$\begin{array}{lccccc}\text { Sim } & 13(38,2) & 3(8,8) & 3(8,8) & - & 19(55,8) \\ \text { Não } & 4(11,8) & 5(14,7) & 5(14,7) & 1(2,9) & 15(44,2) \\ \text { Total } & 17(50,0) & 8(23,5) & 8(23,5) & 1(2,9) & 34^{*}(100,0)\end{array}$

Carga horária diária de trabalho $(\mathrm{p}=0,690)$

$\begin{array}{lccccc}\text { Entre } 8 \text { e }<10 \text { horas } & 4(11,0) & 2(5,6) & - & - & 6(16,7) \\ \text { Entre 10 e <12 horas } & 6(16,7) & 2(5,6) & 2(5,6) & - & 10(27,9) \\ 12 \text { horas ou mais } & 8(22,3) & 5(13,8) & 6(16,7) & 1(2,8) & 20(55,6) \\ \text { Total } & 18(50,0) & 9(25,0) & 8(22,3) & 1(2,8) & 36(100,0) \\ \text { Outro trabalho (p<0,208) } & & & & & \\ \text { Sim } & 15(42,8) & 6(17,2) & 3(8,5) & 1(2,9) & 25(71,4) \\ \text { Não } & 3(8,5) & 3(8,5) & 4(11,5) & - & 10(28,6) \\ \text { Total } & 18(51,4) & 9(25,7) & 7(20,0) & 1(2,9) & 35(100,0)\end{array}$

Renda familiar (salários mínimos**) $(\mathrm{p}=0,592)$

$\begin{array}{lccccc}2 \text { a } 5 & 4(11,8) & 2(5,9) & - & - & 6(17,7) \\ 6 \text { a } 10 & 6(17,6) & 3(8,8) & 2(5,9) & 1(2,9) & 12(35,3) \\ \text { Mais de } 10 & 7(20,6) & 4(11,8) & 5(14,7) & - & 16(47,0) \\ \text { Total } & 17(50,0) & 9(26,5) & 7(20,6) & 1(2,9) & 34^{*}(100,0)\end{array}$

Satisfeito com o programa $(\mathrm{p}=0,609)$

$\begin{array}{lccccc}\text { Sim } & 17(47,2) & 7(19,4) & 7(19,4) & 1(2,8) & 32(88,9) \\ \text { Não } & 1(2,8) & 2(5,6) & 1(2,8) & - & 4(11,1) \\ \text { Total } & 18(50,0) & 9(25,0) & 8(22,3) & 1(2,8) & 36(100,0)\end{array}$

Pensou em desistir do programa $(\mathrm{p}=0,038)$

$\begin{array}{lccccc}\text { Sim } & 6(16,7) & 6(16,7) & 7(19,4) & - & 19(52,8) \\ \text { Não } & 12(33,3) & 3(8,3) & 1(2,8) & 1(2,8) & 17(47,2) \\ \text { Total } & 18(50,0) & 9(25,0) & 8(22,3) & 1(2,8) & 36(100,0)\end{array}$

* As variáveis não respondidas foram excluídas para a análise dos dados.

** Valor do salário mínimo: $R \$ 744,00$.

\section{TABELA 2}

Distribuição dos níveis de depressão segundo características sociodemográficas e de satisfação dos médicos residentes. São José do Rio Preto, 2013-2014

\begin{tabular}{lcccc} 
& \multicolumn{3}{c}{ Depressão } & Total \\
\cline { 2 - 4 } & Ausência & Leve & Moderada & \\
Sexo $(\mathrm{p}=0,292)$ & $\mathrm{n}(\%)$ & $\mathrm{n}(\%)$ & $\mathrm{n}(\%)$ & $\mathrm{n}(\%)$ \\
Masculino & $3(8,6)$ & - & - & $3(8,6)$ \\
Feminino & $17(48,5)$ & $13(37,2)$ & $2(5,7)$ & $32(91,4)$ \\
Total & $20(57,1)$ & $13(37,2)$ & $2(5,7)$ & $35^{*}(100,0)$
\end{tabular}

Faixa etária $(\mathrm{p}=0,001)$

$\begin{array}{lcccc}20-25 \text { anos } & 6(18,8) & 2(6,2) & 1(3,1) & 9(28,1) \\ 26-30 \text { anos } & 12(37,5) & 10(31,3) & - & 22(68,8) \\ 31-35 \text { anos } & - & - & 1(3,1) & 1(3,1) \\ \text { Total } & 18(56,3) & 12(37,5) & 2(6,2) & 32^{*}(100,0) \\ \text { Estado civil }(\mathrm{p}=0,483) & & & & \\ \text { Casado } & 2(5,7) & 3(8,5) & - & 5(14,3) \\ \text { Solteiro } & 18(51,4) & 10(28,6) & 2(5,7) & 30(85,7) \\ \text { Total } & 20(57,1) & 13(37,1) & 2(5,7) & 35^{*}(100,0)\end{array}$

Atividade física $(\mathrm{p}=0,016)$

$\begin{array}{lcccc}\text { Sim } & 13(36,1) & 3(8,3) & - & 16(44,4) \\ \text { Não } & 7(19,4) & 10(27,8) & 3(8,3) & 20(55,6) \\ \text { Total } & 20(55,6) & 13(36,1) & 3(8,3) & 36(100,0) \\ \text { Atividades de lazer }(\mathrm{p}=0,012) & & & \\ \text { Sim } & 14(41,2) & 5(14,7) & - & 19(55,9) \\ \text { Não } & 4(11,8) & 8(23,5) & 3(8,8) & 15(44,1) \\ \text { Total } & 18(53,0) & 13(38,2) & 3(8,8) & 34^{*}(100,0)\end{array}$

Carga horária diária de trabalho $(\mathrm{p}=0,501)$

$\begin{array}{lcccc}\text { Entre } 8 \text { e }<10 \text { horas } & 3(8,3) & 3(8,3) & - & 6(16,6) \\ \text { Entre } 10 \text { e }<12 \text { horas } & 7(19,4) & 3(8,3) & - & 10(27,7) \\ 12 \text { horas ou mais } & 10(27,9) & 7(19,4) & 3(8,3) & 20(55,6) \\ \text { Total } & 20(55,6) & 13(36,1) & 3(8,3) & 36(100,0) \\ \text { Outro trabalho }(\mathrm{p}=0,311) & & & \\ \text { Sim } & 15(42,8) & 9(25,7) & 1(2,9) & 25(71,4) \\ \text { Não } & 5(14,3) & 3(8,5) & 2(5,7) & 10(28,6) \\ \text { Total } & 20(57,1) & 12(34,2) & 3(8,5) & 35^{*}(100,0)\end{array}$

Renda familiar (salários mínimos**) $(\mathrm{p}=0,901)$

$\begin{array}{lcccc}2 \text { a } 5 & 4(11,8) & 2(5,9) & - & 6(17,7) \\ 6 \text { a } 10 & 7(20,6) & 4(11,8) & 1(2,9) & 12(35,3) \\ \text { Mais de } 10 & 8(23,5) & 6(17,6) & 2(5,9) & 16(47,0) \\ \text { Total } & 19(55,9) & 12(35,3) & 3(8,8) & 34^{*}(100,0)\end{array}$

Satisfeito com o programa $(\mathrm{p}=0,432)$

$\begin{array}{lcccc}\text { Sim } & 18(50,0) & 12(33,3) & 2(5,6) & 32(88,9) \\ \text { Não } & 2(5,6) & 1(2,8) & 1(2,8) & 4(11,1) \\ \text { Total } & 20(55,6) & 13(36,1) & 3(8,3) & 36(100,0) \\ \text { Pensou em desistir do programa (p=0,008) } & & \\ \text { Sim } & 6(16,7) & 11(30,5) & 2(5,6) & 19(52,8) \\ \text { Não } & 14(38,9) & 2(5,6) & 1(2,8) & 17(47,2) \\ \text { Total } & 20(55,6) & 13(36,1) & 3(8,4) & 36(100,0)\end{array}$

* As variáveis não respondidas foram excluídas para a análise dos dados.

** Valor do salário mínimo: $R \$ 744,00$. 


\begin{tabular}{|c|c|c|c|c|c|c|c|}
\hline \multicolumn{8}{|c|}{$\begin{array}{l}\text { Relação entre ansiedade e depressão entre os médicos residentes. } \\
\text { São José do Rio Preto, 2013-2014 }\end{array}$} \\
\hline \multicolumn{8}{|c|}{ Depressão } \\
\hline Variáveis & $\begin{array}{c}\text { Ausência } \\
\text { n (\%) }\end{array}$ & $\begin{array}{l}\text { Leve } \\
\mathrm{n}(\%)\end{array}$ & $\begin{array}{c}\text { Moderada } \\
\text { n }(\%)\end{array}$ & $\begin{array}{l}\text { Grave } \\
\text { n (\%) }\end{array}$ & Total & Kappa & p-valor \\
\hline \multicolumn{8}{|l|}{ Ansiedade } \\
\hline Ausência & $15(41,7)$ & $3(8,3)$ & - & - & $18(50,0)$ & \multirow{5}{*}{0,378} & \multirow{5}{*}{0,003} \\
\hline Leve & $4(11,1)$ & $5(13,9)$ & - & - & $9(25,0)$ & & \\
\hline Moderada & $1(2,8)$ & $5(13,9)$ & $2(5,5)$ & - & $8(22,2)$ & & \\
\hline Grave & - & - & $1(2,8)$ & - & $1(2,8)$ & & \\
\hline Total & $20(55,6)$ & $13(36,1)$ & $3(8,3)$ & - & $36(100,0)$ & & \\
\hline
\end{tabular}

Para alguns autores, a participação feminina nas profissões da área da saúde ganhou força com os movimentos feministas, que levaram a mudanças nas relações da mulher com o trabalho ${ }^{15,16}$. Além disso, a prevalência de profissionais do sexo feminino entre os residentes deste estudo pode estar relacionada à afinidade com o cuidado à criança que a especialidade exige. Acredita-se, ainda, que o perfil de profissionais solteiros esteja relacionado com a faixa etária observada, ou seja, profissionais jovens e recém-formados.

A prevalência de ansiedade (50,0\%) e depressão (44,4\%) observada neste estudo foi superior à prevalência de transtornos mentais comuns, encontrada em estudo com residentes médicos e não médicos de diferentes especialidades em Reci$\mathrm{fe}^{12}$. Outro estudo com residentes de Radiologia e diagnóstico por imagem no Rio de Janeiro ${ }^{17}$ encontrou índices de ansiedade semelhantes aos deste estudo, porém a prevalência de depressão entre os residentes cariocas foi maior do que a observada neste estudo. Tais resultados corroboram a ideia de que a organização do trabalho do médico residente nos diferentes programas pode contribuir para o surgimento de índices elevados de transtornos psíquicos, como ansiedade e depressão ${ }^{5,8,9,12}$.

Para Silva et $a l .{ }^{17}$, a falta de determinação e organização dos alunos nos diferentes programas de residência, em associação com os problemas familiares, sociais e econômicos, pode desencadear um processo ansiogênico e/ou depressivo, que culminará com o comprometimento do desempenho acadêmico e profissional dos residentes durante a especialização. Corroborando estes autores, outros estudos mostram que o convívio com problemas de saúde de difícil manejo, o risco de morte dos pacientes, a cobrança dos supervisores, as jornadas de trabalho excessivas são alguns fatores que favorecem o desenvolvimento desses transtornos pelos residentes, especialmente entre os mais jovens ${ }^{4,5,14}$.

Muitas vezes, a longa jornada de trabalho do residente impede que o profissional harmonize sua vida pessoal com as atividades profissionais. Como a Lei 6.932, que regulamenta a residência médica no Brasil, estabelece um regimede 60 horas semanais ${ }^{18}$, muitos profissionais têm prejuízos na realização de atividades de lazer e/ou prática de atividades físicas. Isto potencializa o efeito deletério de fatores como cansaço e estresse inerentes ao trabalho, culminando com prejuízos na saúde física e mental, como o aumento dos níveis de ansiedade e depressão, conforme corroboram os resultados deste estudo.

Além da extensa jornada de trabalho imposta aos residentes pela legislação brasileira, este estudo mostrou que muitos profissionais se dedicam a outra atividade laboral, o que aumenta o risco de desenvolverem transtornos físicos e psicológicos. Fabichaket al. ${ }^{19}$ destacam que o baixo valor da bolsa de estudos recebida pelos residentes leva muitos profissionais a buscarem outras atividades remuneradas, sobrecarregando-se, prejudicando sua qualidade de vida e comprometendo o processo de aprendizagem e a qualidade da assistência prestada aos usuários dos serviços de saúde.

Os níveis de ansiedade e depressão apresentados pelos residentes em Pediatria deste estudo evidenciam que o período de transição aluno-profissional é desgastante, reforçando os resultados de outros estudos com médicos residentes $8,9,12,13,17,19$.

Um estudo com residentes canadenses apontou que a presença de transtornos psicológicos é maior no primeiro ano de formação, quando os profissionais estão em fase de adaptação à nova rotina de trabalho e, por conta disso, apresentam maior insegurança ${ }^{20}$.

Durante aresidência médica, é importante que o profissional consiga administrar os sentimentos negativos relacionados às dificuldades da assistência no sistema de saúde, minimizando o impacto das situações estressoras em sua saúde ${ }^{9}$. Além disso, a implementação de programas de assistência aos residentes pode contribuir para a melhoria do processo de formação e do desenvolvimento das competências profissionais nos diferentes programas de residência médica brasileiros ${ }^{4,5}$. 


\section{CONCLUSÃO}

Embora satisfeitos com o programa, muitos dos residentes de Pediatria avaliados neste estudo já pensaram em desistir da residência. Os índices de ansiedade e depressão encontrados foram superiores aos observados em outros programas, havendo associação destes transtornos com a faixa etária e ausência de atividade física e de lazer, evidenciando a necessidade de maior atenção e suporte aos profissionais.

Os fatores inerentes à atividade laboral dos residentes de Pediatria potencializam o aparecimento desses transtornos entre os profissionais, o que reforça a necessidade de implementar ações de controle dos fatores estressores entre os médicos residentes.

Estes resultados evidenciam que a identificação precoce dos sintomas de ansiedade e depressão entre os residentes de Pediatria pode contribuir para evitar impactos sociais e profissionais causados por esses transtornos, orientando os gestores e supervisores dos programas na viabilização de estratégias de promoção do bem-estar físico e mental desses profissionais.

\section{REFERÊNCIAS}

1. Lima AMC. Residência médica sob a óptica do direito do trabalho. Rev Esmesc. 2010;17(23):175-96.

2. Velho MTAC, Haeffner LB, Santos FG, Silva LC, Weinmann ARM. Residência médica em um hospital universitário: a visão dos residentes. Rev Bras Educ Médica. 2012;36(3):351-7.

3. Nogueira-Martins LA. Cuidando da saúde dos médicos. In: Saúde Integral - A Medicina do Corpo, da Mente e o Papel da Espiritualidade. São Paulo: Senac, 2011. p.67-83.

4. Lourenção LG, Moscardini AC, Soler ZASG. Health and quality of life of medical residents. Rev Assoc Med Bras. 2010; 56(1): 81-90.

5. Dias BA, Pereira MN, Sousa IF, Almeida RJ. Qualidade de vida de médicos residentes de um hospital escola. Sci Med. 2016;26(1):ID22315.

6. Santos FD, Cunha MHF, Rozazzi MLCC, Pedrão JL, Silva LA, Terra FS. O estresse do enfermeiro nas unidades de terapia intensiva adulto: uma revisão da literatura. SMAD, Rev. Eletrônica Saúde Mental Álcool Drog. 2010;6(1):116. Disponível em: http://pepsic.bvsalud.org/scielo. php?script=sci_arttext\&pid=S1806-69762010000100014\&ln $\mathrm{g}=\mathrm{pt} \& \mathrm{t} \operatorname{lng}=$ pt. Acesso em: 10 jun. 2016.

7. Batista FCN, Pawlowytsch PWM. Aspectos emocionais de depressão, ansiedade, desesperança e ideação suicida nos profissionais da unidade de terapia intensiva de um hospital do interior de Santa Catarina. Saúde Meio Ambient. 2012;1(1):188-202.
8. Hoelz L, Campello L. Relação entre Síndrome de Burnout, erro médico e longa jornada de trabalho em residentes de medicina. Rev Bras Med Trab. 2015;13(2):126-34.

9. Silva RM, Goulart CT, Bolzan MEO, Serrano PM, Lopes LFD, Guido La. Stress and hardiness in medical residents. Rev enferm UFPE on line. 2013;7(9):5406-13.

10. Wang YP, Gorenstein C. Psychometric properties of the Beck Depression Inventory-II: a comprehensive review. Rev Bras Psiquiatr. 2013; 35(4):416-31.

11. Gomes-Oliveira MH, Gorenstein C, Lotufo Neto F, Andrade LH, Wang YP. Validation of the Brazilian Portuguese version of the Beck Depression Inventory-II in a community sample. Rev Bras Psiquiatr. 2012; 34(4):389-94.

12. Carvalho CN, Melo-Filho DA, Carvalho JAG, Amorim ACG. Prevalência e fatores associados aos transtornos mentais comuns em residentes médicos e da área multiprofissional. J Bras Psiquiatr. 2013;62(1):38-45.

13. Soares LR, Lopes TMO, Silva MAO, Ribeiro MVA, Almeida Junior MP, Silva RA et al. Burnout e Pensamentos Suicidas em Médicos Residentes de Hospital Universitário. Rev Bras Educ Médica. 2012;36(1):77-82.

14. Rotta DS, Pinto MH, Lourenção LG, Teixeira PR, Gonsalez EG, Gazetta CE. Anxiety and depression levels among multidisciplinary health residents. Rev Rene. 2016;17(3):372-7.

15. Matos IB, Toassi RFC, Oliveira MC. Profissões e ocupações de saúde e o processo de feminização: tendências e implicações. Athenea Digital. 2013;13(2):239-44.

16. Mascarenhas CHM, Prado FO, Fernandes MH, Boery EN, Sena ELS. Qualidade de vida em trabalhadores da área de saúde: uma revisão sistemática. Revista espaço para a saúde. 2014;15(2):72-81.

17. Silva GCC, Koch HA, Sousa EG, Gasparetto E, Buys RC. Anxiety and depression among medical residents in radiology and diagnostic imaging. Rev Bras Educ Médica. 2010;34(2):199-206.

18. Brasil. Lei n. 6.932 de 07 de julho de 1981. Dispõe sobre as atividades do médico residente e dá outras providências. Presidência da República, 1981.

19. Fabichak C, Silva-Júnior JS, Morrone LC. Síndrome de burnout em médicos residentes e preditores organizacionais do trabalho. Rev Bras Med Trab. 2014;12(2):79-84.

20. Hurst C, Kahan D, Ruetalo M, Edwards S. A year in transition: a qualitative study examining the trajectory of first year residents' well-being. BMC Med Educ [Internet]. 2013 [cited 2016 jun 26]; 13:96. Available from: http: / / bmcmededuc.biomedcentral.com/articles/10.1186/1472-6920-13-96. 


\section{CONTRIBUIÇÃO DOS AUTORES}

Luciano Garcia Lourenção contribuiu para a concepção do projeto, análise e interpretação dos dados e redação do artigo. Cláudia Eli Gazetta e Maria Helena Pinto contribuíram para a redação do artigo, revisão crítica relevante do conteúdo intelectual e aprovação final da versão a ser publicada. Priscila Regina Teixeira, Elizangela Gianini Gonzales e Daniela Salvagni Rotta contribuíram com a coleta, análise e interpretação dos dados, e redação do artigo.

\section{CONFLITO DE INTERESSES}

Os autores declaram não haver conflito de interesses.

\section{ENDEREÇO PARA CORRESPONDÊNCIA}

Luciano Garcia Lourenção

Universidade Federal do Rio Grande - Escola de Enfermagem

Rua General Osório, s/nํㅡ - $4^{\circ}$ piso - Centro

Campus da Saúde - Rio Grande

CEP 96201-900 - RS

E-mail: luciano.famerp@gmail.com 